\title{
First reported case of multidrug-resistant Candida auris in Canada
}

\author{
IS Schwartz ${ }^{\star *}$, GW Hammond ${ }^{1}$
}

\section{Abstract}

Candida auris is a fungal pathogen that has recently emerged as a global threat to public health. It was first described in Japan in 2009 and has since been reported in 17 countries on five continents. This case report describes the first reported case of multidrug-resistant $C$. auris in Canada.

In May 2017, a 64 year-old individual was evaluated for chronic otitis externa. Past medical history included a recent hospitalization in India for elective oral surgery that was complicated by an odontogenic brain abscess. Upon return to Canada, the individual was admitted to a hospital for neurosurgical drainage of the brain abscess and parenteral antibiotics. Early during hospitalization, the patient was identified as a carrier of carbapenem-resistant Enterobacteriaceae and was placed on contact precautions. Also early during this hospitalization, a chronic otitis media was managed with placement of a typanostomy tube with drainage of clear fluid from the ear, which continued through the admission and after discharge to a post-neurosurgical rehabilitation facility. During outpatient follow-up, swabs of the ear discharge cultured $C$. auris that was resistant to fluconazole and amphotericin B. There was no clinical response to ototopical antifungal therapy. Surgical evaluation for management of the otomastoiditis is pending.

There is a potential for $C$. auris to cause infection in health care settings. It can persist in hospital environments, has the potential for transmission and can cause invasive disease. It is difficult to identify and often resistant to antifungal medications. The application of infection prevention and control recommendations can help prevent nosocomial transmission. It is now prudent to consider the risk of $C$. auris, in addition to the known risk of other antimicrobial resistant organisms, in any traveller who has been hospitalized while outside the country. When identified, contacting local public health can assist in the tracking and management of this emerging disease.

\begin{abstract}
Affiliation
${ }^{1}$ Section of Infectious Diseases, Departments of Internal Medicine and Medical Microbiology, Max Rady College of Medicine, University of Manitoba, Winnipeg, $\mathrm{MB}$
\end{abstract}

Correspondence: ilan.steven. schwartz@gmail.com

Suggested citation: Schwartz IS, Hammond GW. First reported case of multidrug-resistant Candida auris in Canada. Can Commun Dis Rep. 2017;43 (7/8):150-3. https://doi.org/10.14745/ccdr.v43i78a02

\section{Case}

In May 2017, a 64-year-old individual was evaluated for chronic otitis externa. The patient had a two-year history of recurrent ear complaints. Nine months prior to presentation, a general practitioner clinically diagnosed otomycosis based on bilateral ear pain and drainage of serous "cheesy" fluid from one ear. Topical flumethasone pivalate $0.02 \%$-clioquinol $1 \%$ was prescribed for eight weeks with resolution of the drainage. Two months later, the patient complained of bilateral ear pain without drainage and was prescribed oral amoxicillin by the same clinician for presumed acute otitis media.

Past medical history included oral surgery conducted in India three months prior to presentation, complicated by an odontogenic brain abscess that resulted in a 24-day hospitalization in India. While hospitalized, a high-resolution computed tomography (CT) scan of the temporal bone demonstrated chronic otitis media with mastoiditis, and osteomyelitis of the mastoid, petrous wall and anterior wall of the middle ear cavity on one side and a mild mastoiditis on the other side. The patient was treated with empiric antibacterial and anti-tuberculosis therapy and, after initial clinical improvement, returned to Canada and presented to a community hospital for further care. A CT of the brain demonstrated a persistent left frontal abscess and the patient was transferred to a tertiary care hospital for further investigations and management. Early in the tertiary hospital admission, screening rectal swabs identified carbapenem-resistant Enterobacteriaceae, so the patient was placed on contact precautions. An aspirate of the brain abscess was obtained for diagnosis; although bacterial, mycobacterial and fungal stains and cultures were negative for organisms, amplification and sequencing of 16S RNA identified Streptococcus mitis. The patient was treated with parenteral antibiotics for two months as an inpatient and another month at home, with good clinical and radiographic response. The patient also had a history of emphysema, borderline diabetes mellitus and asymptomatic, early cirrhosis of undetermined etiology.

Shortly after admission to the tertiary hospital in Canada for management of the brain abscess, the patient was also treated by an otolaryngyologist for chronic otitis media with a tympanostomy tube, leading to drainage of clear fluid. The drainage continued throughout the remaining three weeks 
of admission to the tertiary care hospital and a one-month admission for post-neurosurgical rehabilitation in a third hospital for complications arising from the brain abscess.

During outpatient follow-up, a swab of the ongoing ear drainage was sent for bacterial and fungal culture. A yeast grew, identified as Candida auris by matrix-assisted laser desorption ionization-time of flight (MALDI-TOF) mass spectrometry (Bruker MALDI Biotyper System, RUO MBT Compass 4.1.70). Using the CLSI microbroth dilution method, and based on the tentative MIC break-points suggested by the United States (US) Centers for Diseases Control and Prevention (CDC) (1), testing found the $C$. auris sample was resistant to fluconazole and amphotericin $B$, likely resistant to voriconazaole and susceptible to micafungin. (Table 1). The fungus also grew on four repeat swabs of the discharge from the same ear, over a six-week period. Identification of the five isolates as $C$. auris by MALDI-TOF was done by the hospital laboratory. An isolate was sent to a mycology laboratory where drug resistance testing was performed. Whole genome sequence analysis performed by the National Microbiology Laboratory (NML) was consistent with $C$. auris. Further analyses to compare the isolate with global strains are pending.

Table 1: Summary of susceptibility testing of Candida auris isolate from the first reported case in Canada

\begin{tabular}{|c|c|c|c|c|}
\hline \multirow{2}{*}{ Class } & Drug & $\begin{array}{c}\text { Tentative } \\
\text { MIC } \\
\text { Breakpoints }\end{array}$ & $\begin{array}{c}\text { Results } \\
\text { from } \\
\text { C. auris } \\
\text { isolate }\end{array}$ & Interpretation \\
\hline \multirow{2}{*}{ Triazoles } & Fluconazole & $\geq 32 \mu \mathrm{g} / \mathrm{mL}$ & $128 \mu \mathrm{g} / \mathrm{mL}$ & Resistant \\
\cline { 2 - 5 } & Voriconazole & $\mathrm{N}^{2} \mathrm{~A}^{2}$ & $\mathrm{~N} / \mathrm{A}^{2}$ & Likely resistant ${ }^{2}$ \\
\hline Polyenes & $\begin{array}{c}\text { Amphotericin } \\
\mathrm{B}\end{array}$ & $\geq 2 \mu \mathrm{g} / \mathrm{mL}$ & $2 \mu \mathrm{g} / \mathrm{mL}$ & Resistant \\
\hline Echinocandins & Micafungin & $\geq 4 \mu \mathrm{g} / \mathrm{mL}$ & $0.5 \mu \mathrm{g} / \mathrm{mL}$ & Susceptible \\
\hline
\end{tabular}

Abbreviations: MIC, minimum inhibitory concentration, N/A, Not available

Tentative (MIC) breakpoints for $C$. auris from the United States Centers for Disease Control and Prevention (CDC) (1)

2 Until MIC breakpoint is available, CDC suggests considering using fluconazole susceptibility as a surrogate for second generation triazole susceptibility assessment, noting however that isolates that are resistant to fluconazole may respond to other triazoles occasionally

Upon outpatient reassessment by an otolaryngologist, there was evidence of myringitis and otitis externa. The ear canal contained moist white debris, which was removed. A tympanostomy tube was in situ, draining a clear discharge; this tube was left in place. The patient was retreated topically with flumethasone pivalate $0.02 \%$-clioquinol $1 \%$ drops, but without improvement after two weeks. The patient has been referred to a neurotologist for surgical assessment. Case resolution of the otitis externa and the chronic mastoiditis was pending at the time of the report.

\section{Discussion}

This is the first reported case of multidrug-resistant $C$. auris in Canada. C. auris was first described in Japan in 2009 (3), and has since been reported in 17 countries on five continents $(2,4)$. Only four C. auris isolates from prior to 2009 were identified retrospectively among 15,271 isolates in a global candidemia registry (5), suggesting rapid global dispersal. C. auris has already established a firm foot-hold in some healthcare settings. In developing countries, the risk of $C$. auris transmission is compounded by limitations in capacity for fungal identification, antifungal susceptibility testing and infection prevention and control (IPC). In India, for instance, C. auris already accounts for $5 \%$ of the isolates implicated in candidemia in intensive care units nationwide (6) and as many as $30 \%$ in some centres (7).

Several features of $C$. auris make infection challenging to manage and heighten concern for nosocomial transmission: it can persist on patients and in hospital environments; it is difficult to identify; it can cause invasive disease; and treatment options are limited $(1,4,7)$. Indeed, recalcitrant healthcare-associated outbreaks have been reported from the United Kingdom and the United States $(9,10)$, and genetic studies have implicated closely related (clonal) strains, suggesting effective horizontal transmission $(5,7,9,10)$.

\section{C. auris can persist in hospital environments}

The tenacity of $C$. auris has been demonstrated during outbreak investigations in which the organism could be isolated from patients' skin (up to three months following infection) and patient environments $(9,14)$. The capacity of $C$. auris to adhere to polymeric surfaces and form biofilms may contribute to difficulty in eradicating this organism from the environment (15).

\section{C. auris is difficult to identify}

Correct identification of $C$. auris can be difficult for clinical microbiology laboratories and this may delay implementation of appropriate IPC procedures. C. auris can be misidentified by commercial identification systems such as Vitek-2 (as C. haemulonii or C. famata) and API-20C (as Rhodotorula glutinis, C. sake or Saccharomyces cerevisiae) (16). C. auris can be correctly identified by MALDI-TOF using "research-use only" databases and by sequencing of the internal transcribed spacer and D1-D2 domains (16). Limitations also exist for some antifungal susceptibility testing methods (16); for instance, Vitek-2 may give falsely elevated MICs for amphotericin B and the broth microdilution method may give falsely elevated MICs for caspofungin (16).

\section{C. auris can cause invasive disease}

Although the first reported cases of $C$. auris were patients with chronic otomycosis $(3,11)$, most subsequent cases have been healthcare-associated and involved candidemia or other invasive infections (2). Persistent candidemia (lasting up to three weeks after antifungal initiation) and high mortality rates have been observed (7). A murine model of candidemia suggested that C. auris was nearly as virulent as $C$. albicans, the predominant cause of invasive candidiasis worldwide (12); precise virulence factors are still being investigated, although some appear to be strain-dependant (13).

\section{Treatment options are limited}

Decreased antifungal susceptibility among isolates is a universal feature of C. auris. Fluconazole resistance exceeds $90 \%(5)$ and reduced susceptibility to voriconazole may approach $50 \%$ (2). Tentative breakpoints for the newer triazoles, posaconazole and isavuconazole, are not available, but low MICs suggest that they may have activity against $C$. auris (2). Resistance to amphotericin $B$ occurs in up to $35 \%$ of isolates (5) and echinocandin resistance has been reported in $2-8 \%$ of isolates $(2,5)$. Resistance to all three major classes of antifungals was reported in $4 \%$ of isolates (5). 
The optimal management of $C$. auris disease is currently unknown (2). Where invasive disease is involved, systemic antifungal therapy should be guided by results of susceptibility testing. Management of otomycosis and otomastoiditis caused by $C$. auris is even less clear. Evaluations of the efficacy of ototopical agents against $C$. auris have not been published and the options for multidrug-resistant isolates, as in this case, are limited.

\section{Infection prevention and control can limit spread}

Infection prevention and control measures can be effective in limiting the spread of C.auris. Interim IPC recommendations from the CDC include single rooms and contact precautions for colonized or infected patients, periodic reassessments for ongoing colonization as well as daily and terminal cleaning of surfaces with a disinfectant effective against Clostridium difficile spores (See text box) (10).

\section{Summary of Candida auris Interim Recommendations for Healthcare Facilities from the United States Centers for Disease Control and Prevention (10)}

\section{Use standard and contact precautions}

Patients in acute care hospitals and nursing homes should be placed in single rooms on Standard and Contact Precautions. Continue Contact Precautions as long as the person is colonized with C.auris.

\section{Use a disinfectant effective against Clostridium difficile spores}

Healthcare facilities that have patients with $C$. auris infection or colonization should ensure thorough daily and terminal cleaning and disinfection of these patients' rooms with hospital-grade disinfectant effective against Clostridium difficile spores

\section{Conduct periodic reassessments}

Periodic reassessments for presence of $C$. auris colonization (every 1-3 months) can help inform duration of infection control measures. Assessments of colonization should involve testing of, at a minimum, swabs of the axilla and groin and also may include sites yielding $C$. auris on previous cultures.

\section{Provide notification upon transfer}

When patients are transferred to other healthcare facilities, receiving facilities should receive notification of $C$. auris infection or colonization and the level of precautions recommended.

\section{Document two negative tests for $C$. auris before stopping infection control procedures}

Two or more assessments performed at least one week apart with negative results are needed before discontinuing infection control precautions. The patient or resident should not be on antifungal medications active against $C$. auris at the time of these assessments (wait one week).

\section{Conclusion}

This patient is currently clinically stable and awaiting surgical assessment. Best practices for IPC are being carried out in the three hospitals where this patient was cared for, local public health has been informed and regional IPC follow up is underway. Fortunately, as part of the treatment of the brain abscess associated with hospitalization outside the country, routine screening for antimicrobial resistant organisms found carbapenem-resistant Enterobacteriaceae and the patient had been placed on contact precautions.

The source of $C$. auris is not yet known, but one source could have been related to the hospitalization in India where $C$. auris is known to be endemic $(6,17)$. Due to the chronicity of ear symptoms and the prior (unconfirmed) clinical diagnosis of otomycosis, the infection may have preceded the patient's most recent travel; however, the patient had been in India prior to the onset of the ear symptoms as well. Genotyping to identify the most likely source of the infection is pending.

As with other antimicrobial-resistant organisms (18), travel-associated infection or colonization from $C$. auris is likely to be increasingly encountered by clinicians. In the United States, two cases of $C$. auris infection were observed in returning travellers who had been hospitalized abroad $(10,14)$. In addition, in a multi-state hospital-associated outbreak in the US, C. auris isolates from New York and New Jersey were highly related to one another and were similar to South Asian isolates (10); this suggests the introduction of $C$. auris acquired abroad to healthcare facilities in these states. Anyone who has been hospitalized while outside of the country may be at increased risk for antimicrobial-resistant organisms, including C. auris; contact precautions and testing may be indicated. This approach may help contain $C$. auris from nosocomial transmission within and between Canadian healthcare facilities. Alerting local public health can also assist in efforts to track and manage this emerging disease.

\section{Authors' statement}

ISS - Conceptualization, Data Collection, Writing - Original Draft, Review and Editing

GWH - Conceptualization, Investigation, Data Collection, Writing - Review and Editing, and Supervision

\section{Conflict of Interest}

None.

\section{Acknowledgements}

The authors gratefully acknowledge the patient who provided written consent for publication of this case report, Dr. Arnold Frohlich for his expert clinical opinion and for review of the manuscript, microbiologists and technologists at Diagnostic Services Manitoba, Cadham Provincial Laboratory and the National Microbiology Laboratory. We thank Dr. Eric Bow and Dr. Kelly MacDonald for helpful discussions regarding case management. The authors also wish to acknowledge the editorial staff of CCDR and anonymous reviewers for expeditious evaluation of this Rapid Communication. 


\section{References}

1. Centers for Disease Control and Prevention (CDC). Candida auris Interim Recommendations for Healthcare Facilities and Laboratories [Internet]. [cited 2017 Jun 23]. Available from: https://www.cdc.gov/fungal/diseases/candidiasis/ recommendations.html.

2. Chowdhary A, Sharma C, Meis JF. Candida auris: A rapidly emerging cause of hospital-acquired multidrug-resistant fungal infections globally. PLoS Pathog 2017 May;13(5):e1006290. DOI: http://dx.doi.org/10.1371/journal.ppat.1006290. PubMed (https://www.ncbi.nlm.nih.gov/entrez/query.fcgi?cmd=Retrieve\& $\mathrm{db}=$ PubMed\&list_uids=28542486\&dopt=Abstract).

3. Satoh K, Makimura K, Hasumi Y, Nishiyama Y, Uchida K, Yamaguchi H. Candida auris sp. nov., a novel ascomycetous yeast isolated from the external ear canal of an inpatient in a Japanese hospital. Microbiol Immunol 2009 Jan;53(1):41-4. DOI: http://dx.doi.org/10.1111/j.1348-0421.2008.00083.x. PubMed (https://www.ncbi.nlm.nih.gov/entrez/query.fcgi?cmd=Retrieve\& $\mathrm{db}=$ PubMed\&list_uids=19161556\&dopt=Abstract).

4. Al-Siyabi T, Al Busaidi I, Balkhair A, Al-Muharrmi Z, Al-Salti M, Al'Adawi B. First report of Candida auris in Oman: clinical and microbiological description of five candidemia cases. J Infect 2017 Jun;4453(17)1-3:S0163-4453(17)30164-0. 10.1016/j.jinf.2017.05.016. PubMed (https://www.ncbi.nlm. nih.gov/entrez/query.fcgi? $\mathrm{cmd}=$ Retrieve $\& \mathrm{db}=$ PubMed\&lis t_uids=28579303\&dopt=Abstract).

5. Lockhart SR, Etienne KA, Vallabhaneni S, Farooqi J, Chowdhary A, Govender NP et al. Simultaneous Emergence of MultidrugResistant Candida auris on 3 Continents Confirmed by Whole-Genome Sequencing and Epidemiological Analyses. Clin Infect Dis 2017 Jan;64(2):134-40. DOI: http://dx. doi. org/10.1093/cid/ciw691. PubMed (https://www.ncbi.nlm. nih.gov/entrez/query.fcgi?cmd=Retrieve\&db=PubMed\&lis t_uids=27988485\&dopt=Abstract).

6. Rudramurthy SM, Chakrabarti A, Paul RA, Sood P, Kaur $\mathrm{H}$, Capoor MR et al. Candida auris candidaemia in Indian ICUs: analysis of risk factors. J Antimicrob Chemother 2017 Jun;72(6):1794-801. DOI: http://dx.doi.org/10.1093/jac/dkx034. PubMed (https://www.ncbi.nlm.nih.gov/entrez/query.fcgi?cmd= Retrieve\&db=PubMed\&list_uids=28333181\&dopt=Abstract).

7. Chowdhary A, Sharma C, Duggal S, Agarwal K, Prakash A, Singh PK et al. New clonal strain of Candida auris, Delhi, India. Emerg Infect Dis [Internet]. Centers for Disease Control and Prevention; 2013 Oct [cited 2017 Jun 27];19(10):1670-3. Available from: http://www.ncbi.nlm.nih.gov/pubmed/24048006.

8. Clancy CJ, Nguyen MH. Emergence of Candida auris : An International Call to Arms. Clin Infect Dis [Internet]. 2017 Jan 15 [cited 2017 Jun 22];64(2):141-3. Available from: http://www.ncbi. nlm.nih.gov/pubmed/27989986.

9. Schelenz S, Hagen F, Rhodes JL, Abdolrasouli A, Chowdhary A, Hall A et al. First hospital outbreak of the globally emerging Candida auris in a European hospital. Antimicrob Resist Infect Control 2016 Oct;5(1):35-42. DOI: http://dx.doi.org/10.1186/ s13756-016-0132-5. PubMed (https://www.ncbi.nlm.nih. gov/entrez/query.fcgi?cmd=Retrieve \&db=PubMed\&list_ uids $=27777756 \&$ dopt=Abstract).

10. Tsay S, Welsh RM, Adams EH, Chow NA, Gade L, Berkow EL et al.; MSD. Notes from the Field: Ongoing Transmission of Candida auris in Health Care Facilities - United States, June 2016-May 2017. MMWR Morb Mortal Wkly Rep
2017 May;66(19):514-5. DOI: http://dx.doi.org/10.15585/ mmwr.mm6619a7. PubMed (https://www.ncbi.nlm.nih. gov/entrez/query.fcgi?cmd=Retrieve\&db=PubMed\&list_ uids=28520710\&dopt=Abstract).

11. Kim MN, Shin JH, Sung H, Lee K, Kim EC, Ryoo N et al. Candida haemulonii and Closely Related Species at 5 University Hospitals in Korea: Identification, Antifungal Susceptibility, and Clinical Features. Clin Infect Dis [Internet]. 2009 Mar 15 [cited 2017 Jun 22];48(6):e57-61. Available from: http://www.ncbi.nlm.nih.gov/ pubmed/19193113.

12. Ben-Ami R, Berman J, Novikov A, Bash E, Shachor-Meyouhas Y, Zakin S et al. Multidrug-Resistant Candida haemulonii and C. auris, Tel Aviv, Israel. Emerg Infect Dis 2017 Feb;23(1):195-203. PubMed (https://www.ncbi.nlm.nih.gov/entrez/query.fcgi?cmd= Retrieve\&db=PubMed\&list_uids=28098529\&dopt=Abstract).

13. Larkin E, Hager C, Chandra J, Mukherjee PK, Retuerto M, Salem I et al. The Emerging Pathogen Candida auris: Growth Phenotype, Virulence Factors, Activity of Antifungals, and Effect of SCY-078, a Novel Glucan Synthesis Inhibitor, on Growth Morphology and Biofilm Formation. Antimicrob Agents Chemother [Internet]. 2017 May [cited 2017 Jun 23];61(5):e02396-16. Available from: http://www.ncbi.nlm.nih. gov/pubmed/28223375.

14. Vallabhaneni S, Kallen A, Tsay S, Chow N, Welsh R, Kerins J et al. Investigation of the First Seven Reported Cases of Candida auris, a Globally Emerging Invasive, Multidrug-Resistant FungusUnited States, May 2013-August 2016. Am J Transplant 2017 Jan;17(1):296-9. DOI: http://dx.doi.org/10.1111/ajt.14121. PubMed (https://www.ncbi.nlm.nih.gov/entrez/query.fcgi?cmd= Retrieve\&db=PubMed\&list_uids=28029734\&dopt=Abstract).

15. Sherry L, Ramage G, Kean R, Borman A, Johnson EM, Richardson MD et al. Biofilm-Forming Capability of Highly Virulent, Multidrug-Resistant Candida auris. Emerg Infect Dis 2017 Feb;23(2):328-31. DOI: http://dx.doi.org/10.3201/ eid2302.161320. PubMed (https://www.ncbi.nlm.nih. gov/entrez/query.fcgi?cmd=Retrieve\&db=PubMed\&list_ uids $=28098553 \&$ dopt $=$ Abstract).

16. Kathuria S, Singh PK, Sharma C, Prakash A, Masih A, Kumar A et al. Multidrug-Resistant Candida auris Misidentified as Candida haemulonii: Characterization by Matrix-Assisted Laser Desorption Ionization-Time of Flight Mass Spectrometry and DNA Sequencing and Its Antifungal Susceptibility Profile Variability by Vitek 2, CLSI Broth Microdilution, and Etest Method. J Clin Microbiol 2015 Jun;53(6):1823-30. DOI: http:// dx.doi.org/10.1128/JCM.00367-15. PubMed (https://www.ncbi. $\mathrm{nlm}$.nih.gov/entrez/query.fcgi?cmd=Retrieve\&db=PubMed\&li st_uids $=25809970 \&$ dopt $=$ Abstract).

17. Chowdhary A, Anil Kumar V, Sharma C, Prakash A, Agarwal $\mathrm{K}$, Babu R et al. Multidrug-resistant endemic clonal strain of Candida auris in India. Eur J Clin Microbiol Infect Dis 2014 Jun;33(6):919-26. DOI: http://dx.doi.org/10.1007/ s10096-013-2027-1. PubMed (https://www.ncbi.nlm.nih. gov/entrez/query.fcgi?cmd=Retrieve\&db=PubMed\&list uids $=24357342 \&$ dopt=Abstract).

18. MacFadden DR, Bogoch II, Brownstein JS, Daneman N, Fisman $D$, German $\mathrm{M}$ et al. A passage from India: Association between air traffic and reported cases of New Delhi Metallo-betalactamase 1 from 2007 to 2012. Travel Med Infect Dis [Internet]. 2015 Jul [cited 2017 Jun 24];13(4):295-9. Available from: http:// linkinghub.elsevier.com/retrieve/pii/S1477893915001040. 